\title{
Implementation of a pilot electronic parent support tool in and after neonatal intensive care unit discharge
}

\author{
Joanne Lagatta ${ }^{1}{ }^{凶}$, Margaret Malnory ${ }^{1}$, Elizabeth Fischer ${ }^{1}$, Mary Davis ${ }^{2}$, Patti Radke-Connell ${ }^{1}$, Cheryl Weber ${ }^{2}$ and Susan Cohen iD $^{1}$
}

(c) The Author(s), under exclusive licence to Springer Nature America, Inc. 2021

OBJECTIVE: To describe an electronic parent support tool for the neonatal intensive care unit (NICU), and to assess whether support requests changed with staff availability.

METHODS: We implemented secure text- or email-based parent support in the NICU and in the week after discharge. Questionnaires asked whether a parent would like psychology, social work, child life, chaplain, or post-discharge nurse support. Requested referrals were placed, and customized online resources and contacts were provided. We assessed whether requests changed based on in-person resource availability.

RESULTS: Of 378 infants in our NICU from May to December, 202 parents agreed to participate. The proportion agreeing to participate increased over time (38-59\%, $p=0.012)$. Post-discharge nurse requests decreased over time $(90-45 \%, p=0.033) ;$ other requests did not change significantly.

CONCLUSIONS: An electronic tool increased parent support availability in the NICU and following discharge, even after staff were available at the bedside.

Journal of Perinatology (2022) 42:1110-1117; https://doi.org/10.1038/s41372-021-01303-3

\section{BACKGROUND}

The neonatal intensive care unit (NICU) setting presents significant challenges to parents facing recovery from a high-risk pregnancy, difficult birth experience, and critical illness of their infant [1-8]. Increasingly we also understand the risks of perinatal mental health concerns. Both mothers and fathers are vulnerable to depression and anxiety in the perinatal period, with increased rates of distress for parents of children hospitalized in the NICU [9-13]. Additionally, parents may then face the stress of caring for their child's complex medical needs after discharge. As the medical field has recognized the challenges of parents in the NICU setting, psychosocial support has increased to include social work, case managers, chaplains, child life, and psychology, whose expertize is designed to mitigate these stressors and meet the mental health needs of infants and families. Despite this, parents' needs may often get lost while caring for a critically ill child [14]. Since parent health and access to resources are crucial for child well-being, better systems to support parents in the NICU have the potential to improve the lives of both parents and children [7].

Like most hospitals, at the beginning of the COVID-19 pandemic Children's Wisconsin added stricter limits on the number of allowed visitors [15]. Families were restricted first to one parent or caregiver per child through June, then two parents or caregivers per child. At the same time, support staff including social work, psychology, chaplains, and child life were asked to refrain from attending rounds or being at the bedside unless called or consulted by a nurse or provider. Since remote staff included those who are essential to supporting parent well-being in the NICU, we were concerned that these changes would lead to unmet parent needs. In addition, we were concerned that reduced parent visitation due to both hospital visitor restrictions and school-closure-associated childcare demands would result in unmet teaching needs after an infant's NICU discharge. In response, we designed and implemented a multidisciplinary secure text- or email-based system of checking in with parents in the NICU and in the week after discharge. As staff returned to working in-person, we continued offering the electronic check-ins. This allowed us to continue offering families support while assessing next steps for our NICU in terms of outreach and follow-up.

Our objectives were to describe infant and parent characteristics associated with the use of an electronic parent support tool in the NICU and in the week following discharge, and to assess whether requests for support changed based on the availability of in-person staff resources.

\section{METHODS}

This was a single-center prospective pilot project starting in May 2020, designated exempt from human subjects review by the Children's Wisconsin Institutional Review Board. The project was conducted in the Children's Wisconsin NICU, a 70-bed level IV single-bed NICU with a fetal consult coordinating center attached to a delivery hospital. There are no designated sections of higher versus lower acuity beds in this unit; any infant was eligible for family participation regardless of illness or acuity.

Parents who spoke English and were anticipated to have custody of their child after NICU discharge were eligible to be offered this service. Language criteria were used because of the text nature of our pilot outreach. Custody criteria were used because of the goal to reach parents post-discharge caring for their babies; in addition, parents with custody

${ }^{1}$ Department of Pediatrics, Medical College of Wisconsin, Milwaukee, WI, USA. ${ }^{2}$ Children's Wisconsin, Milwaukee, WI, USA. ${ }^{\circledR}$ email: jlagatta@mcw.edu 
concerns automatically receive more intensive support in our unit. We identified these families on manual chart review by the documentation already present from social work, psychology and nursing. Drug screens were not automatic grounds for exclusion. Parents were contacted by telephone by a nurse on our team; we called up to three times before designating that family as unavailable for contact. Alternatively, parents could also opt to participate by indicating their interest on an informational form included in their child's admission paperwork. Any parent could opt to participate, whether married or not. Multiple parents were not excluded from each participating, but no families asked for multiple caregivers to enroll.

For those who opted to participate, parents were asked whether they wanted to receive notifications via text or email. The first contact from our team was a test message, verifying contact information before proceeding. After verifying contact information, we sent a total of three questionnaires as a link to a REDCap questionnaire; the link was sent via text or email per parent preference [16]. The questionnaires were developed with input from our nursing, case management, social work, psychology, chaplain, and child life teams, and reviewed for literacy by a communications specialist. The first was sent immediately after enrollment; it asked whether a parent would like psychologist consultation or online resources, social work contact via phone or email, child life support or online resources, or chaplain support. The second was sent one week later for parents of infants still in the NICU, asking whether their needs had changed and if they required additional support. Parents requesting online resources were automatically directed to appropriate links. Requests for consultation were reviewed daily by our team and directed to appropriate NICU staff.

The third questionnaire was sent within $48-72 \mathrm{~h}$ of infant discharge, after review of the infant chart for health record details including appointments, equipment, and care team members. The questionnaire asked whether a parent had questions regarding feeding and breastfeeding, home medical equipment, upcoming appointments, general infant care questions, help managing stress or anxiety, or other concerns. Parents with questions regarding feeding and breastfeeding, appointments, and home medical equipment were given customized information regarding who to call for support, populating contact information from the child's discharge summary. Parents with concerns about managing general infant care issues were referred to online resources with anticipatory guidance; they were also provided contact information and encouraged to contact their primary care physician. Parents with concerns managing stress, sadness or anxiety were provided links to community and online resources; all parents were offered phone contact from a psychologist in our NICU. All responses were reviewed daily by our team; medical questions were relayed to NICU nurses who routinely call families after discharge to ensure appropriate follow-up. At the end of each questionnaire, we confirmed the phone and email contacts that we had available. If the post-discharge questionnaire was not completed, we checked the clinical records for more updated information. In all questionnaires, we asked parents for feedback to improve this pilot service and allowed an open-ended response. We called parents who indicated a willingness to discuss the program to identify their perspectives and suggestions for improvement.

In addition to discharge details from the infant's health record, we recorded additional details from the health record to understand which families were using this pilot service; these details included inborn, gender, gestational age, birth weight, surgery during hospitalization, mechanical ventilation, length of stay in our NICU, maternal age, maternal race and ethnicity, and maternal postpartum depression screening score using the Edinburgh Postpartum Depression Questionnaire [17, 18].

\section{Statistical analysis}

We first compared infant and parent characteristics between eligible parents who did or did not opt to enroll in our pilot service, and between parents who enrolled but did or did not respond to questionnaires. For parents who responded to questionnaires, we evaluated which infant or parent characteristics were associated with resource requests; we also compared the proportion of resource requests over time to determine whether specific needs changed as our staffing and visitation improved. To compare differences over time, we grouped admissions into time periods based on staff availability and potential differences in family logistic challenges: (1) May, the first month of enrollment, when many staff were working remotely and visitors were limited to one per infant; (2) JuneAugust, when visitors increased to two per infant, and staff began returning to in-person availability; (3) September-December, when there were no further staffing changes; parents with older children may have been juggling in-person and remote schooling. To assess how our outreach resulted in number of actual services delivered, we counted documentation of social work and chaplain contacts in the six months prepandemic (9/1/19-3/1/20), 1 month pre-project (4/1/20-5/1/20), and six months into the project $(6 / 1 / 20-1 / 1 / 21)$; psychology contacts are not always documented to maintain parent confidentiality. Differences in proportions were compared with chi-squared or Fisher's exact tests differences in medians were compared with Kruskal-Wallis tests. Trends over time were assessed with Mantel-Haenszel tests. Documentation counts were presented as counts/month and counts/month/admission, and examined descriptively. STATA version 16 (STATACorp, College Station, TX) was used for analyses. A $p$ value of $<0.05$ was used to indicate statistical significance.

\section{RESULTS}

Of a total of 399 patients cared for in our NICU during the study period, we attempted to contact 378 eligible infant-parent dyads. Of the 21 categorized as ineligible for outreach, four were due to custody concerns, 11 language, two infant death, and four anticipated transfer prior to discharge. We were able to reach 239 (63\%) parents; 202 agreed to participate $(85 \%$ of those reached; $53 \%$ of eligible). Figure 1 shows proportions of parentinfant dyads eligible, contacted, and enrolled by study period. When grouped by study period (first month of enrollment, summer, and fall), over $50 \%$ of eligible parents agreed to participate at all study periods $(85-90 \%$ of those who we reached by phone) except for the first month of enrollment, when many parents indicated that this would have been helpful earlier in their NICU stay but was no longer needed at the time we launched the pilot. There were no significant differences over time in proportion of those reached by phone $(p=0.442)$, but significant increases over time in the proportion who agreed to participate both overall $(p=0.012)$ and among those who were reached by phone ( $p=$ $0.005)$.

Of the 202 parents who were interested in participating, 105 (52\%) responded to the inpatient questionnaires. Of the 202 participating parents, 186 infants were discharged from the NICU; $81(44 \%)$ responded to a home questionnaire. Table 1 displays characteristics of eligible infant-parent dyads. 105 parents

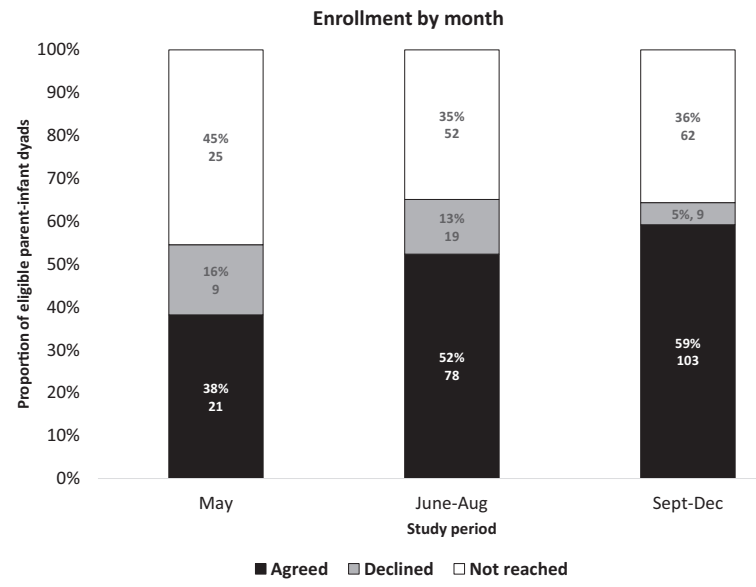

Fig. 1 Enrollment in a pilot electronic parent support tool over time. Figure 1 displays changes over study periods in proportion of parents who agreed, declined, and could not be reached for participation: May, first month of pilot project, one parent per child, most staff working remotely; June-August, two parents per child, staff transitioning back to bedside; September-December, two parents per child, staff back to bedside. We noted a statistically significant increase in the proportion of parents agreeing to participate over time as assessed by Mantel-Haenszel chi-squared test for trend, both overall $(p=0.012)$ and as a proportion of those reached by phone $(p=0.005)$. 
Table 1. Participation and questionnaire completion characteristics.

\begin{tabular}{|c|c|c|c|c|}
\hline \multirow[t]{2}{*}{ Variable } & \multirow[t]{2}{*}{ All eligible } & \multicolumn{3}{|c|}{ Agreed to participate } \\
\hline & & No & Yes & $p$ \\
\hline$n$ & 378 & 176 & 202 & \\
\hline Inborn & $232(61 \%)$ & $109(62 \%)$ & $123(61 \%)$ & 0.975 \\
\hline $\begin{array}{l}\text { Gestational } \\
\text { age (weeks) }\end{array}$ & $35(32-38)$ & 37 (33-39) & $34(31-37)$ & $<0.001$ \\
\hline $\begin{array}{l}\text { Birth } \\
\text { weight }(\mathrm{kg})\end{array}$ & $2.5(1.7-3.2)$ & $2.9(1.9-3.4)$ & $2.2(1.5-2.9)$ & $<0.001$ \\
\hline $\begin{array}{l}\text { Ever } \\
\text { intubated }\end{array}$ & 129 (34\%) & $61(35 \%)$ & 68 (34\%) & 0.839 \\
\hline $\begin{array}{l}\text { Surgery } \\
\text { in NICU }\end{array}$ & 93 (25\%) & $40(23 \%)$ & $53(26 \%)$ & 0.429 \\
\hline $\begin{array}{l}\text { LOS } \\
\text { NICU (days) }\end{array}$ & $16(5-38)$ & $9(2-28)$ & $20(10-42)$ & $<0.001$ \\
\hline $\begin{array}{l}\text { Maternal age } \\
\text { (years) }\end{array}$ & $30(26-33)$ & $30(25-34)$ & $30(26-33)$ & 0.906 \\
\hline $\begin{array}{l}\text { Race/ } \\
\text { ethnicity }\end{array}$ & & & & 0.666 \\
\hline Black & 77 (21\%) & $32(18 \%)$ & $45(22 \%)$ & \\
\hline White & 197 (52\%) & 96 (55\%) & $101(50 \%)$ & \\
\hline Asian & $14(4 \%)$ & $8(5 \%)$ & $6(3 \%)$ & \\
\hline Hispanic & $21(6 \%)$ & $8(5 \%)$ & $13(6 \%)$ & \\
\hline $\begin{array}{l}\text { Other/ } \\
\text { unknown }\end{array}$ & $69(18 \%)$ & $32(18 \%)$ & 37 (18\%) & \\
\hline PPD > 14 & $22(6 \%)$ & $11(6 \%)$ & $11(5 \%)$ & 0.886 \\
\hline
\end{tabular}

Characteristics of the sample: all eligible infants, then those who did or did not agree to participate at initial contact. There were no significant differences in these characteristics between parents who agreed to participate who did versus did not respond to the NICU or home questionnaires (data not shown).

NICU Neonatal intensive care unit, LOS Length of stay, PPD $>14$ a postpartum depression screening score of $>14$ on the Edinburgh Postpartum Depression Scale (scores $>14$ prompt psychology or social work referral).

Values are proportions or medians and interquartile ranges, as appropriate; $p$ values indicate chi-squared, Fisher's exact, or Kruskal-Wallis tests. Bold highlights $p$ values with values $<0.05$.

responded to the NICU questionnaire, and 81 responded to the home questionnaire. Infants of parents who agreed to participate were of younger gestational age, lower birth weight, and had a longer NICU stay. No infant or parent characteristics distinguished between parents who agreed to participate who did versus did not respond to the inpatient or home questionnaire; response rates did not differ based on text versus email method of delivery.

Table 2 displays the questions and response details. Of parents who responded to the inpatient questionnaire, 22 (21\%) reported moderate or significant difficulty managing their feelings of sadness, anxiety, and loneliness; 27 ( $26 \%$ of total; $66 \%$ of those with other children) reported that the siblings of the hospitalized infant were having issues with anxiety, jealousy or loneliness. There were 23 requests for social work consultation, 20 requests for chaplain support, 29 requests for psychology resources, and 8 requests for child life resources. Among parents responding to the home questionnaire, $45(56 \%)$ had a question that required information or follow-up; 38 (47\%) were referred for a nurse call, and 17 (21\%) were referred for a psychologist call. For needs referred for nursing followup, $23(28 \%)$ had questions about routine infant care, and $22(27 \%)$ had questions about feeding and breastfeeding. Of parents with medical equipment and appointment questions, the most common questions were related to feeding equipment or gastrostomy-tuberelated follow-up appointments.
Table 3 displays characteristics of responding parents who did versus did not request resources. In the NICU, psychology resource requests were more common among parents of infants with smaller birth weight, who were ever intubated or who required surgery in the NICU. Chaplain requests were more common among parents of infants with a longer NICU stay. No infant or parent characteristics distinguished those who requested social work support. The proportion of responding parents who requested resources was not significantly different across study periods. After NICU discharge, nursing call requests were more common among parents of infants born at an earlier gestational age, lower birth weight, and longer NICU length of stay; the proportion of nursing requests decreased over the study periods. Characteristics that were not significant between groups included inborn, gestational age, race/ethnicity, need for discharge equipment, or number of specialist appointments. All resource requests were manually received by our team and relayed via secure message to the appropriate staff for follow-up, with the parent's preferred contact information and available time; in all cases, staff members communicated with us to confirm that the request had been received and contact made. Counts of documented contacts from social work and chaplains pre-COVID, pre-outreach, and during this project are presented in Fig. 2. There was a reduction in monthly contacts at the beginning of the pandemic compared to pre-pandemic baseline, which increased after beginning our outreach project to near-baseline levels.

In general, both the inpatient and post-discharge questionnaires were well received by staff and parents. Parents' suggestions for additional electronic communication included a better record of members of a large care team, especially when most communication happens via phone rather than in person; better access to clinical status updates such as oral feeding and respiratory support changes; communication about transportation or parking issues that might change daily in the event of construction; a ready list of resources available that might be missed if a NICU tour could not happen in the first day of an infant's stay; more systematic asking about community resource needs and social support at home. Staff chaplains, social workers, and psychologists commented that it was helpful to have access to additional contact information that helped them connect with families that they had been previously unable to reach. The nurses calling families after discharge commented that they appreciated knowing families' specific concerns prior to a phone call to direct their conversation.

\section{DISCUSSION}

Our goal was to describe infant and parent characteristics associated with the use of an electronic parent support tool in the NICU and in the week following discharge, and to assess whether requests for support changed based on the availability of in-person staff resources. We found the that overall proportion of parents enrolling in our pilot service increased over time, even as staff were increasingly available at the bedside. Earlier gestational age, lower birth weight, and longer NICU length of stay were associated with more parent requests for inpatient and outpatient support.

Electronic access to health care has become a high priority. Many systems have accelerated their efforts in telehealth to increase access to non-emergent care during the COVID-19 pandemic [19-21]. Further, the 21st Century Cures Act has increased system efforts to make electronic health records more accessible to patients and families [22, 23]. Unlike these major system changes, our REDCap text-based questionnaire system was neither telehealth nor embedded in the electronic health record. Advantages to our approach were the speed and low cost of setup; our project began because our original vision for telehealthenabled NICU follow-up was delayed due to system constraints. 
Table 2. Questionnaire responses.

INPATIENT QUESTIONNAIRE $(n=105)$

It is normal to feel more sadness, anxiety, or loneliness with a baby in the NICU. How would you describe your experience managing your feelings of sadness, anxiety, or loneliness during this time?

We have psychologists on staff who help take care of parents of babies in the NICU. Would you like any support from a psychologist?

Social workers can provide emotional support. They may have ideas about financial or other resources to help you through this challenging time. Would you like a social worker to contact you?

It is common for older siblings of babies in the NICU to have feelings of anxiety, jealousy, or sadness with a big life change. How are your other kids doing?

Child life specialists can help suggest ways to help older siblings cope with a baby in the NICU. We can also provide developmentally appropriate education to help siblings understand a new diagnosis. Can we help you with any of the following?

We have chaplains who offer emotional, spiritual or religious support according to your values and beliefs. Chaplains are available both in person and by phone. Would you like any support?

\section{HOME QUESTIONNAIRE $(n=81)$}

We are so glad that your child is home with you! How can we help you adjust? Check all that you might need help with:

Feeding a newborn infant is hard, especially a baby who has been in the NICU. Which of these questions can we help with? Choose all that apply.

Coming home from the NICU is both exciting and scary. Being home feels better than being in the hospital, but the team that was there for you in the NICU feels very far away. Would you like one of our psychologists to help connect you with some support?

Indicated any need for information or follow-up

Had a question that was referred to nursing

Had a question referred to psychology

Inpatient and home questionnaires and responses. All parents received these starting questions, with actual wording and response choices edited slightly for brevity. For most questions, parents were able to choose more than one response, so percentages do not add up to $100 \%$. For the home questionnaire, parents with questions about feeding, equipment and appointments received customized follow-up directed to their infant's particular discharge needs. Rather than detail each potential question and response, the last three rows indicate the number of parents with questions that were referred for followup calls.

NICU neonatal intensive care unit.

This "lower-tech" approach has mixed advantages and disadvantages for families. Text rather than video limits the applicability to families with lower health literacy, but not every family has access to reliable internet to support video clinic visits as compared to the $97 \%$ of families in the world with access to a smart phone [24-27]. Similarly, patient portals directly connected to the electronic health record would allow more direct communication with members of the health care team; however, participants may be more likely to respond to a text message than log onto a patient portal [27-29]. In that context, it was interesting that an increasing

\section{Options}

n (\%)

No problems

$35(34 \%)$

$37(45 \%)$

$17(16 \%)$

$5(5 \%)$

$21(20 \%)$

$8(8 \%)$

$10(10 \%)$

$16(15 \%)$

7 (7\%)

$17(16 \%)$

36 (34\%)

41 (39\%)

$21(20 \%)$

Prayer or blessing said for my child

Already working with a chaplain

Options

Feeding and breastfeeding

Appointments

Baby care questions

Managing my stress, sadness or nxiety

Need to talk to lactation consultant

$6(7 \%)$

How do I know if my baby is eating

How long can my baby go between feeding?

$4(5 \%)$

My baby keeps spitting up.

I would like a call from a psychologist . 


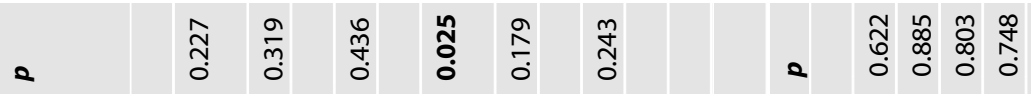

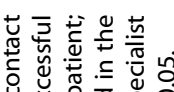

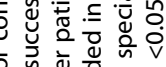

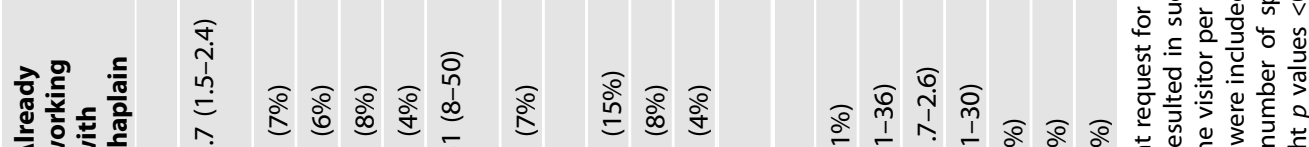

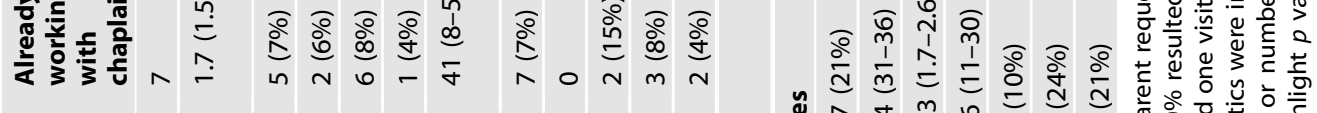

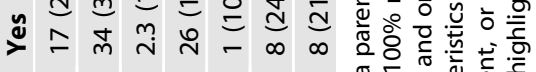

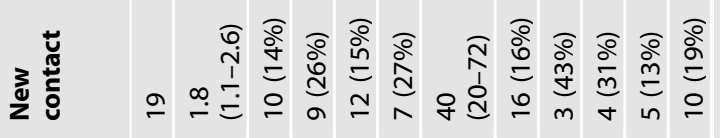

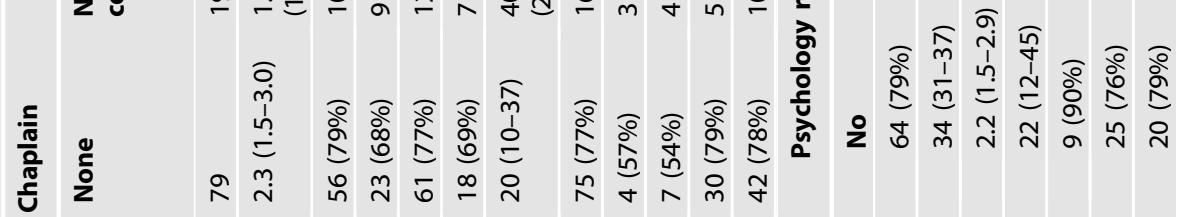
윽춰

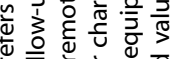

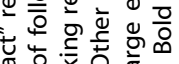

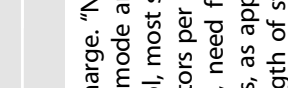

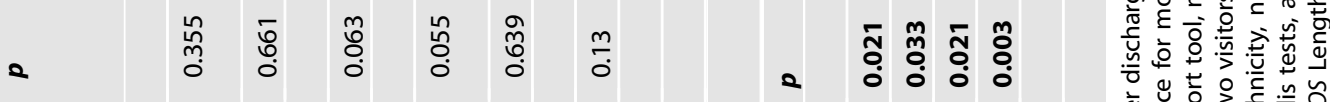

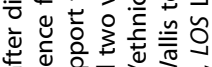

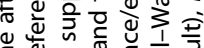

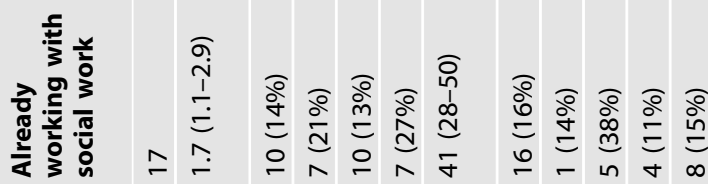

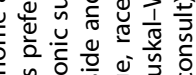

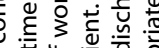

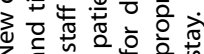

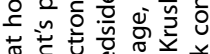

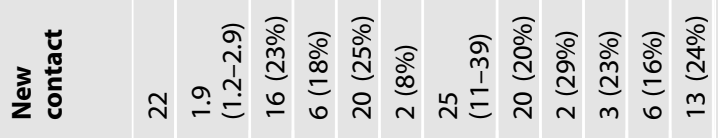

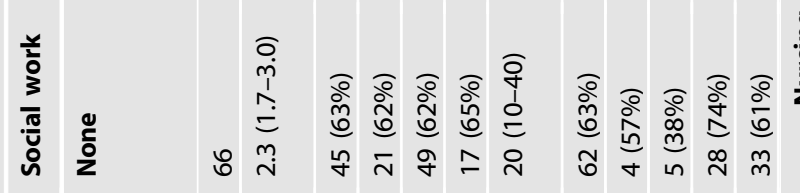

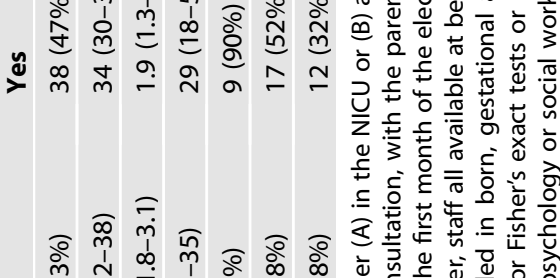

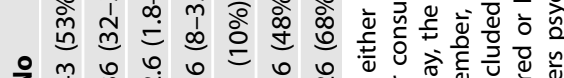

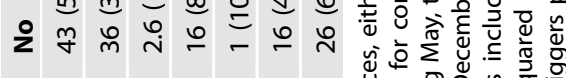

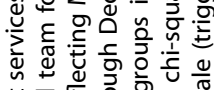

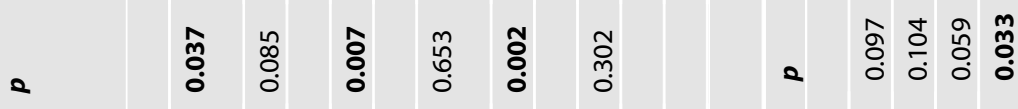

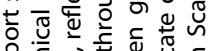

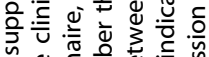

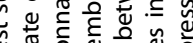

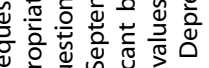

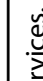

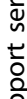

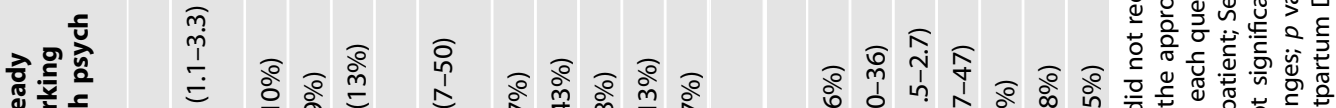

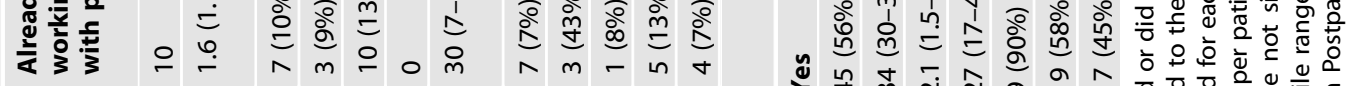

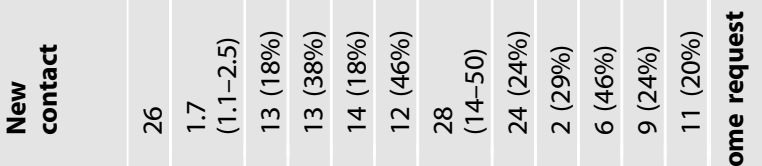

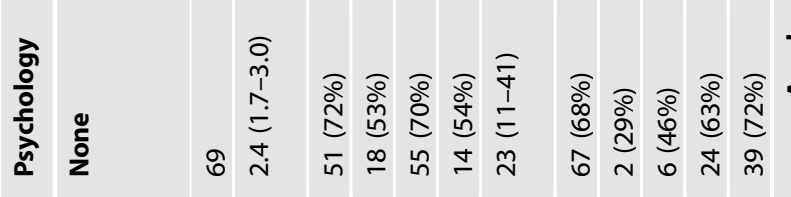

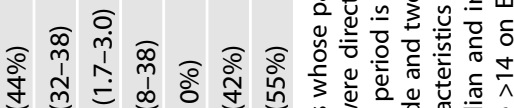
ע

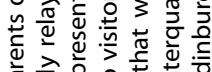

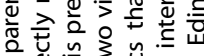

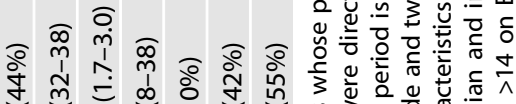

$\frac{\partial}{2}$

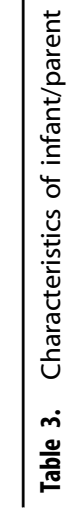

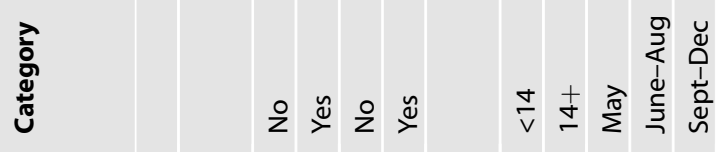

i⿱

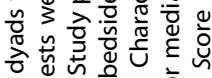

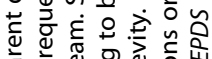
인<smiles>[Te]C1CCCCC1</smiles><smiles>C1CCCCC1</smiles>

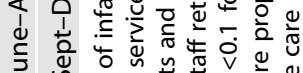

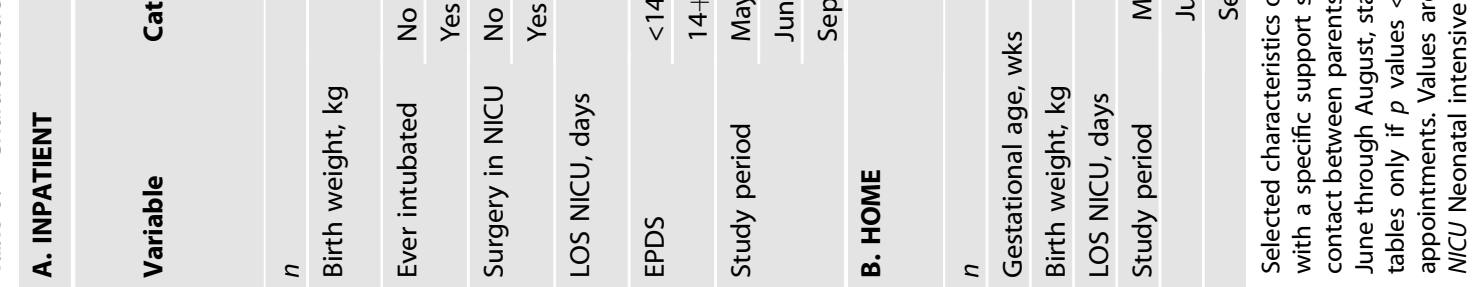




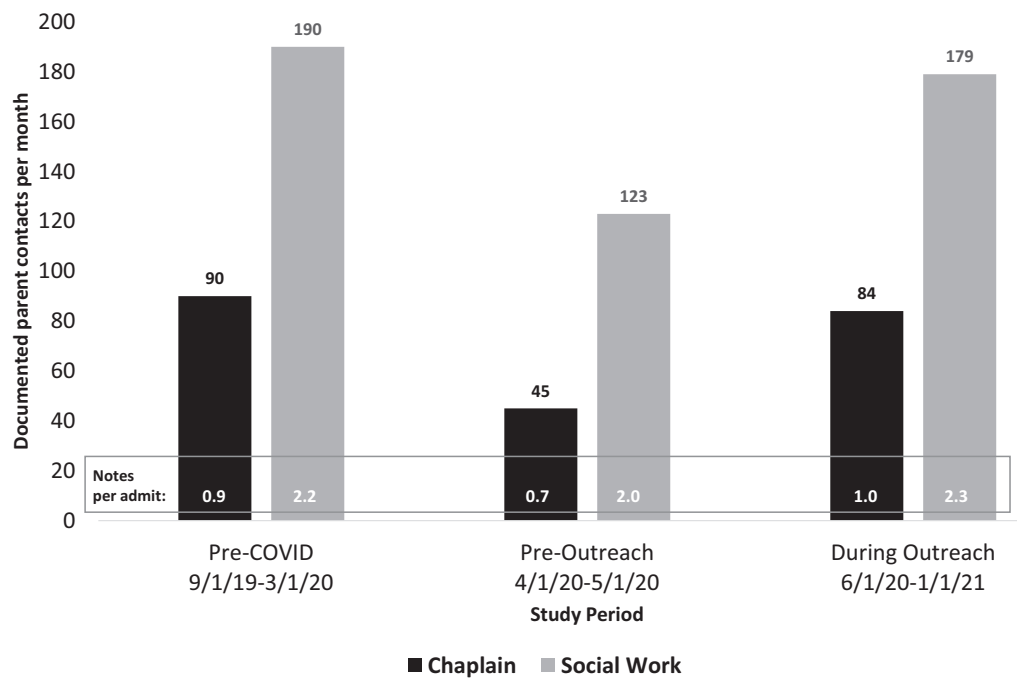

Fig. 2 Parent contacts per month before and during outreach. Figure 2 displays the number of parent contacts per month before and during this project, in 3 periods: "pre-COVID, 9/1/19-3/1/20", for a baseline number of documented social work and chaplain contacts per month; "pre-outreach, 4/1/20-5/1/20," the period during the pandemic during which the project was not yet up and running; "During Outreach 6/1/20-1/1/21", which was the first 6 full months of our project. Black bars indicate counts per month of chaplain documented contacts, and gray bars indicate counts per month of social work documented contacts. The box labeled "notes per admit" and white numbers overlying each bar indicates the number of documented contacts per month, divided by the number of admissions, to estimate how the contact count was affected by changes in census. Results are reported as descriptive rather than using statistical comparison, since by the nature of social work and chaplain communication, this represents a count of contact rather than the confidential content of a given communication which is unavailable in the electronic health record.

Supporting parents' needs in the NICU requires a broad range of expertize. We were able to place additional requests for psychology consultation both in the inpatient and outpatient settings. Although parents with higher scores on the Edinburgh Postpartum Depression scale were more likely to be working with a psychologist already, new requests were most common among parents with lower postpartum depression scores, reinforcing that screening alone does not detect the entirety of parents' needs. These findings highlight opportunities to increase the availability of mental health resources for parents in the NICU and after discharge [30]. Our own NICU has started screening for anxiety in addition to postpartum depression; $[2,5,31]$ based on these findings we are also working toward incorporating electronic methods of mental health screening to supplement our current model of bedside nurses doing the screening and referring for psychology consultation. We are also in the process of incorporating psychologists into our neonatal follow-up clinic to increase access to post-discharge mental health support. Similarly, we were able to place additional requests for social work and chaplain support, which were not more frequent when our staff were working remotely as opposed to their usual daily accessibility in the NICU, and not associated with a specific infant or parent characteristics. As hospitals like ours consider how to optimize the use of electronic health tools, allowing parents direct access to request support for themselves may be an area for future exploration.

Preparing parents for their infants' home health needs requires significant nursing and case management effort during the NICU stay; multi-faceted individualized approaches have been identified as a key factor in successful discharge planning leading to reduced outpatient healthcare utilization [32-41]. After discharge, we found that requests for support were more common among infants with earlier gestational age and longer NICU length of stay, although nursing requests decreased over time as parent visiting and in person outpatient clinic visits resumed. A one-time electronic outreach is certainly less comprehensive than many transition home programs that have been shown to reduce readmissions and promote better health of preterm infants $[32,40]$. At the same time, securing comprehensive follow-up for infants after the NICU is a significant challenge in many parts of the country [42-44]. Our own center is working to adopt remote care strategies as part of our strategy to improve our neonatal follow-up program.

Strengths of our study include the availability of data over a period of several months including visitor and staffing changes, which allowed us to get a broader sense of the potential impact of electronic outreach strategies, the multidisciplinary tool development, and parent and staff feedback. Weaknesses that limit generalizability include the single-center nature of the project, the English-only and text-only availability at this stage. Reaching $63 \%$ of parents in the first days after NICU admission is lower than we had hoped. We were limited by non-clinical staff being restricted from visiting the bedside, such that we were not able to confirm contact information in person. We also did not restrict eligibility by infant length of stay, such that some families were discharged before we were able to reach them. While we were able to count the number of documented social work and chaplain contacts to assess the impact of our outreach, we were not able to assess the quality of that contact. Nurses, social workers, psychologists, and chaplains commented that it was helpful to have an idea of parents' concerns and best modes of contact prior to initiating communication, but that is not feasible to measure by chart review. In future research and quality improvement efforts to improve the value of NICU follow-up, it will be important to measure explicitly how an intervention impacts the use of preventive and acute healthcare utilization.

In summary, the use of a text- and email-based electronic parent support tool increased the availability of parent support services in the NICU and in the week following discharge, and was utilized to access resources both while staff and parents were remote and allowed at the bedside. As efforts to increase electronic access to health care accelerate, maintaining direct connections between parents and needed resources has the potential to improve the health of both parents and children. 


\section{REFERENCES}

1. Bakewell-Sachs S, Gennaro S. Parenting the post-NICU premature infant. MCN Am J Matern child Nurs. 2004;29:398-403.

2. Carter JD, Mulder RT, Darlow BA. Parental stress in the NICU: the influence of personality, psychological, pregnancy and family factors. Personal Ment Health. 2007;1:40-50.

3. Enlow E, Herbert SL, Jovel IJ, Lorch SA, Anderson C, Chamberlain LJ. Neonatal intensive care unit to home: the transition from parent and pediatrician perspectives, a prospective cohort study. J Perinatol. 2014;34:761.

4. Herzer M, Godiwala N, Hommel KA, Driscoll K, Mitchell M, Crosby LE, et al. Family functioning in the context of pediatric chronic conditions. J developmental Behav pediatrics: JDBP. 2010;31:26-34.

5. Malin KJJT, McAndrew S, Westerdahl J, Leuthner J, Lagatta J, editor Infant Illness Severity and the development of parent posttraumatic stress disorder after the neonatal intensive care unit. Advances in Neonatal Care. 2019;140:104930.

6. McAndrew S, Acharya K, Westerdahl J, Brousseau DC, Panepinto JA, Simpson P, et al. A prospective study of parent health-related quality of life before and after discharge from the neonatal intensive care unit. J Pediatrics. 2019;213:38-45.e3.

7. Msall ME. Promoting parenting supports and engagement for infants born preterm. The J Pediatrics. 2019;210:10-12.

8. Singer LT, Salvator A, Guo S, Collin M, Lilien L, Baley J. Maternal psychological distress and parenting stress after the birth of a very low-birth-weight infant. JAMA 1999;281:799-805.

9. O'Hara MW, McCabe JE. Postpartum depression: current status and future directions. Annu Rev Clin Psychol. 2013;9:379-407.

10. Fairbrother N, Young AH, Janssen P, Antony MM, Tucker E. Depression and anxiety during the perinatal period. BMC Psychiatry. 2015;15:206.

11. Paulson JF, Bazemore SD. Prenatal and postpartum depression in fathers and its association with maternal depression: a meta-analysis. Jama 2010;303:1961-9.

12. Mental health trajectories of fathers following very preterm birth: associations with parenting [press release]. United Kingdom: Oxford University Press 2020.

13. Mounts KO. Screening for maternal depression in the neonatal ICU. Clin Perinatol. 2009;36:137-52.

14. Hynan MT, Steinberg Z, Baker L, Cicco R, Geller PA, Lassen S, et al. Recommendations for mental health professionals in the NICU. J perinatology: Off J Calif Perinat Assoc. 2015;35:S14-8.

15. Darcy Mahoney A, White RD, Velasquez A, Barrett TS, Clark RH, Ahmad KA. Impact of restrictions on parental presence in neonatal intensive care units related to coronavirus disease 2019. J perinatology: Off J Calif Perinat Assoc. 2020;40:36-46.

16. Project REDCap [Available from: https://www.project-redcap.org/.

17. Thombs BD, Benedetti A, Kloda LA, Levis B, Riehm KE, Azar M, et al. Diagnostic accuracy of the Edinburgh Postnatal Depression Scale (EPDS) for detecting major depression in pregnant and postnatal women: protocol for a systematic review and individual patient data meta-analyses. BMJ Open. 2015;5:e009742.

18. Cox J, Holden J, Sagovsky R. Detection of postnatal depression. development of the 10-item edinburgh postnatal depression scale. Br J Psychiatry: J Ment Sci. 1987;150:782-6.

19. Hong Y-R, Lawrence J, Williams D Jr, Mainous lii A. Population-level interest and telehealth capacity of US hospitals in response to COVID-19: cross-sectional analysis of google search and national hospital survey data. JMIR Public Health Surveill. 2020;6:e18961.

20. Hron JD, Parsons CR, Williams LA, Harper MB, Bourgeois FC. Rapid Implementation of an Inpatient Telehealth Program during the COVID-19 Pandemic. Appl Clin Inf. 2020;11:452-9.

21. Wosik J, Fudim M, Cameron B, Gellad ZF, Cho A, Phinney D, et al. Telehealth transformation: COVID-19 and the rise of virtual care. J Am Med Inform Assoc. 2020;27:957-62.

22. Pageler NM, Webber EC, Lund DP. Implications of the 21 st century cures act in pediatrics. Pediatrics 2021;147:e2020034199.

23. Gabay M. 21st Century Cures Act. Hosp Pharm. 2017;52:264-5.

24. Dol J, Delahunty-Pike A, Anwar Siani S, Campbell-Yeo M. eHealth interventions for parents in neonatal intensive care units: a systematic review. JBI Database Syst Rev Implement Rep. 2017;15:2981-3005.

25. Lee SH, Nurmatov UB, Nwaru BI, Mukherjee M, Grant L, Pagliari C. Effectiveness of mHealth interventions for maternal, newborn and child health in low- and middle-income countries: Systematic review and meta-analysis. J Glob Health. 2016;6:010401.

26. Richardson B, Dol J, Rutledge K, Monaghan J, Orovec A, Howie K, et al. Evaluation of mobile apps targeted to parents of infants in the neonatal intensive care unit: systematic app review. JMIR Mhealth Uhealth. 2019;7:e11620.

27. Orr T, Campbell-Yeo M, Benoit B, Hewitt B, Stinson J, McGrath P. Smartphone and internet preferences of parents: information needs and desired involvement in infant care and pain management in the NICU. Adv Neonatal Care. 2017;17:131-8.
28. Hopkins IG, Dunn K, Bourgeois F, Rogers J, Chiang VW. From development to implementation-A smartphone and email-based discharge follow-up program for pediatric patients after hospital discharge. Healthcare 2016;4:109-15.

29. Olivia Kim U, Barnekow K, Ahamed SI, Dreier S, Jones C, Taylor $M$, et al. Smartphone-based prenatal education for parents with preterm birth risk factors. Patient Educ counseling. 2019;102:701-8.

30. McGowan EC, Du N, Hawes K, Tucker R, O'Donnell M, Vohr B. Maternal mental health and neonatal intensive care unit discharge readiness in mothers of preterm infants. The J pediatrics. 2017;184:68-74.

31. Spitzer RL, Kroenke K, Williams JB, Lowe B. A brief measure for assessing generalized anxiety disorder: the GAD-7. Arch Intern Med. 2006;166:1092-7.

32. Liu Y, McGowan E, Tucker R, Glasgow L, Kluckman M, Vohr B. Transition home plus program reduces medicaid spending and health care use for high-risk infants admitted to the neonatal intensive care unit for 5 or more days. The. J Pediatrics. 2018;200:e3.

33. Hospital discharge of the high-risk neonate-proposed guidelines. American academy of pediatrics. committee on fetus and newborn. Pediatrics 1998;102:411-7.

34. Berger SP, Holt-Turner I, Cupoli JM, Mass M, Hageman JR. Caring for the graduate from the neonatal intensive care unit. At home, in the office, and in the community. Pediatr Clin North Am. 1998;45:701-12.

35. Berry JG, Ziniel SI, Freeman L, Kaplan W, Antonelli R, Gay J, et al. Hospital readmission and parent perceptions of their child's hospital discharge. Int J Qual Health Care: J Int Soc Qual Health Care. 2013;25:573-81.

36. Fetus Co, Newborn. Hospital Discharge of the High-Risk Neonate. Pediatrics. 2008;122:1119.

37. Lakshmanan A, Kubicek K, Williams R, Robles M, Vanderbilt DL, Mirzaian CB, et al. Viewpoints from families for improving transition from NICU-to-home for infants with medical complexity at a safety net hospital: a qualitative study. BMC pediatrics. 2019;19:223.

38. Raffray M, Semenic S, Osorio Galeano S, Ochoa, Marín SC. Barriers and facilitators to preparing families with premature infants for discharge home from the neonatal unit. Perceptions of health care providers. Investigacion y educacion en enfermeria. 2014;32:379-92.

39. Smith VC, Hwang SS, Dukhovny D, Young S, Pursley DM. Neonatal intensive care unit discharge preparation, family readiness and infant outcomes: connecting the dots. J Perinatol. 2013;33:415.

40. Spinner SS, Girifalco RB, Gibson E, Stavis RL, Greenspan JS, Spitzer AR. Earlier discharge of infants from neonatal intensive care units: a pilot program of specialized case management and home care. Delaware Valley Child Health Alliance. Clin pediatrics. 1998;37:353-7.

41. Vohr B, McGowan E, Keszler L, O'Donnell M, Hawes K, Tucker R. Effects of a transition home program on preterm infant emergency room visits within 90 days of discharge. J perinatology: Off J Calif Perinat Assoc. 2018;38:185-90.

42. Hintz SR, Gould JB, Bennett MV, Gray EE, Kagawa KJ, Schulman J, et al. Referral of very low birth weight infants to high-risk follow-up at neonatal intensive care unit discharge varies widely across California. J Pediatrics. 2015;166:289-95.

43. Swearingen C, Simpson P, Cabacungan E, Cohen S. Social disparities negatively impact neonatal follow-up clinic attendance of premature infants discharged from the neonatal intensive care unit. J perinatology: Off J Calif Perinat Assoc. 2020;40:790-7.

44. Harmon SL, Conaway M, Sinkin RA, Blackman JA. Factors associated with neonatal intensive care follow-up appointment compliance. Clin pediatrics. 2013;52:389-96.

\section{ACKNOWLEDGEMENTS}

The authors gratefully acknowledge Angie Biersach, Sandy Dykstra, Jane Fryda, Kim Gajewski, Sally Keller, Haley Koenke, Lauren Kostich, Courtney Rademann, and Kristin Ross for their contributions to this project.

\section{AUTHOR CONTRIBUTIONS}

$\mathrm{JL}$ was responsible for study concept and design, leading design of the parent support tool used in this study, leading data analysis, and writing the first draft of the manuscript. MM was responsible for data collection. PRC contributed to data management and analysis and review of the manuscript. EF, MD, CW and SC were responsible for content contributions to the design of the parent support tool and review of data. All authors reviewed and contributed to the manuscript and approve the final version as submitted. 
FUNDING

Supported by K23 HL136525 (to JL) and an award from the Children's Research Institute at the Medical College of Wisconsin (to JL). This work was supported by the National Institutes of Health (Grant Number K23HL136525 [to JL]) and a Children's Research Institute award (to JL).

\section{COMPETING INTERESTS}

The authors declare no competing interests.

\section{ADDITIONAL INFORMATION}

Correspondence and requests for materials should be addressed to Joanne Lagatta.

Reprints and permission information is available at http://www.nature.com/ reprints

Publisher's note Springer Nature remains neutral with regard to jurisdictional claims in published maps and institutional affiliations. 